African Crop Science Journal by African Crop Science Society is licensed under a Creative Commons Attribution 3.0 Uganda License. Based on a work at www.ajol.info/ and www.bioline.org.br/cs DOI: http://dx.doi.org/10.4314/acsj.v25i1.4

\title{
INFLUENCE DES ENGINS ET TECHNIQUES DE PECHE SUR L'ABONDANCE RELATIVE DES ESPECES DANS LA BASSE VALLEE DE L'OUEME AU BENIN
}

A.H. ATTINGLI, S. AHOUANSOU MONTCHO, E.W. VISSIN ${ }^{1}$, L.H. ZINSOU et P.A. LALEYE Laboratoire d'Hydrobiologie et d'Aquaculture, Faculté des Sciences Agronomiques, Université d'AbomeyCalavi, 01BP 526 Cotonou, Bénin

${ }^{1}$ Laboratoire Pierre PAGNEY Climat, Eau, Ecosystème et Développement, BP 526 Cotonou, Bénin Auteur correspondant: hermasattingli@yahoo.fr

(Received 21 September, 2016; accepted 17 February, 2017)

\section{RÉSUMÉ}

Les pêcheries dans la Basse Vallée de l'Ouémé (BVO) sont soumises à d'énormes pressions de pêche. L'analyse de l'influence des engins et techniques de pêche sur le stock de poisson est un moyen de mise en évidence de ces pressions. Cette étude vise à fournir des informations nécessaires à une exploitation durable des ressources halieutiques dans le milieu. La collecte des données a été effectuée de Mai 2015 à Mai 2016 sur 16 zones de pêcheries. Les indices de nuisance et d'impact des engins et techniques de pêche ainsi que l'abondance relative des espèces ont été calculés en utilisant le logiciel XLSTAT. Une cartographie de ces paramètres a été faite par interpolation linéaire sous le logiciel ArcGis 9.3. Les résultats ont révélé 8 engins et techniques de pêche. "Acadja" et filet maillant sont les plus utilisés dans les zones de pêcherie situées en aval et au centre de la Vallée (Tu: 0,350 à 0,502 ) et ont un indice de nuisance élevé (IN: 0,209 chacun) sur l'ichtyofaune. L'influence des engins et techinques de pêches (II: 0,126 à 0,146 ) affecte aussi l'abondance relative des espèces de poissons (AR: 2,410 à 3,723). L'impact des engins et techniques de pêche a une influence positive significative, mais localisée, sur l'abondance relative des espèces $\left(\mathrm{r}=0,849 ; \mathrm{R}^{2}=72,20 \% ; \mathrm{F}=36,28 ; \mathrm{P}=3,12^{*} 10^{-5}\right)$. Des études complémentaires relatives à la pêche expérimentale sont nécessaires pour l'élaboration d'un plan de gestion durable des zones de pêcheries de la basse vallée de L’Ouémé.

Mots Clés: Engins et techniques de pêche, Indices de nuisance, chtyofaune, Basse Vallée de l'Ouémé, abondance relative

\begin{abstract}
The fishing areas in the lower Valley of Ouémé (BVO) in Benin are subject to huge fishing pressure. Fishing gears and techniques used in this valley could be influencing fish populations. The objective of this study was to analyse the influence of fishing gears and techniques on fish population to provide information for sustainable management of fish resources in the area. Data were collected from May 2015 to May 2016 in 16 fishing areas. The harm indexes and impacts of gears and fishing techniques, as well as the relative species' abundance were calculated using XLSTAT Software. Ordinary kriging technique in ArcGis 9.3 Software was used to map the measured parameters. Results revealed eight gears and fishing techniques. "Acadja" and gillnet were the most used gears in the downstream and centre of the fishing areas (Tu: 0.350 to 0.502 ) since they showed high harm index (IN: 0.209 each) on the ichthyofauna. The influence of fishing techniques and tools (II: 0.126 to 0.146 ) influenced relative abundances (AR: 2.410 to 3.7230) of fish species. The effect of gears and fishing techniques
\end{abstract}


had a significant but localised positive influence on the relative species' abundance $\left(r=0.857 ; \mathrm{R}^{2}=72.20 \% ; \mathrm{F}=\right.$ 34.56; $\left.\mathrm{P}=3.12 * 10^{-5}\right)$. Further studies are needed to develop a plan for sustainable management of the fisheries in the lower Valley of Ouémé.

Key Words: Fishing gears and techniques, harm index, ichthyofauna, Oueme valley, relative abundance

\section{INTRODUCTION}

Le Bénin est un pays à vocation essentiellement agricole, où l'agriculture, l'élevage et la pêche représentent $49 \%$ de l'activité économique (MAEP, 2009). La quantité de poissons fournie représente environ $28,5 \%$ de la consommation des protéines animales dans le pays (FAO, 2006) et correspond à la majeure partie des protéines d'origine animale des populations tout en leur garantissant une source importante de revenue. La Basse Vallée de l'Ouémé (BVO), partie inférieure du fleuve Ouémé $(510 \mathrm{Km})$ joue un rôle important dans le chapelet de plans et cours d'eau qui alimentent cette économie dans le pays.Elle regorge en effet d'importants écosystèmes favorables à un peuplement de plus de 87 espèces (Lalèyè et al., 2004; Chikou, 2006), pour 122 espèces recensées sur l'ensemble du fleuve selon les mêmes auteurs. Plus de 7816 acteurs de pêche et 18931 pirogues (Direction des Pêches, 2008) ont été dénombrés dans la BVO qui connait par ailleurs une importante croissance démographique (INSAE, 2013).

Mais depuis quelques années, les besoins des populations en ressources halieutiques ont accrus alors que les prises ont chuté de $15 \%$ (MAEP, 2009). Ce qui serait dû à une baisse des rendements de pêche. Le rendement des principaux engins de pêche en effet est en baisse : 2,2 $\mathrm{kg}$ de poissons par jour de pêche pour le filet maillant, $3,5 \mathrm{~kg}$ pour le filet dobah et $1,4 \mathrm{~kg}$ pour le filet épervier contre respectivement 5,2 4,3 et 1,6 kg obtenus au cours des années 1968-69 (Lalèyè et al., 2007). La baisse de rendement peut être aussi attribuée à la dégradation des conditions environnementales des écosystèmes aquatiques présents dans ce secteur (Attingli et al., 2016; Zinsou et al., 2016).

Pour faire face aux changements globaux qui affectent les écosystèmes aquatiques, des progrès considérables sont enregistrés à l'échelle mondiale dans les techniques de repérage, de captures et de conditionnement des ressources halieutiques (Murawski, 2000). Mais des progrès qui constituent en même temps un facteur de surexploitation des plans d'eau.

l'histoire récente de la pêche fait état des niveaux d'exploitation particulièrement élevée et d'exemples spectaculaires de surexploitation des stocks (Balirwa et al., 2003; Kantoussan, 2007). $30 \%$ des stocks de poissons mondiaux sont surexploités, $57 \%$ sont exploités à la limite supérieure de leur productivité et seulement $13 \%$ ne sont pas pleinement exploités (FAO, 2011). Or, la surexploitation peut conduire à une réduction de la biodiversité, ou dans certains cas, à l'extinction d'espèces ou de groupes d'espèces (Kantoussan, 2007). De nos jours, la plupart des zones de pêche subissent une très forte pression de pêche et de nombreuses espèces cibles sont surexploitées (Worn et al., 2006). Dans le delta central du Niger, Laë (1992) a relevé une intensité de la surexploitation des pêcheries.

Eu égard à la prépondérance de l'outillage encore à l'étape artisanale dans l'exploitation des plans et cours d'eaux au Bénin et en Afrique, la documentation de l'impact des engins et techniques de pêche sur l'ichtyofaune est une approche qui pourrait contribuer à la gestion efficiente et durable des écosystèmes aquatiques. Ce qui justifie la présente étude qui fournit des informations sur le degré de nuisance des engins et techniques de pêche sur l'ichtyofaune de la BVO ainsi que leur 
influence sur la distribution des espèces dans le milieu.

\section{MÉTHODOLOGIE}

Milieu d'étude. La Basse Vallée de l'Ouémé (BVO) est située dans la partie inférieure du fleuve Ouémé (plus grand bassin fluvial du Bénin). Elle est constituée du moyen et du bas delta de l'Ouémé (Chikou, 2006) et regroupe 4 Communes (Bonou, Adjohoun, Dangbo et Aguégués). La BVO s' étend entre $6^{\circ} 24^{\prime}$ $6^{\circ} 52^{\prime}$ de latitude Nord et $2^{\circ} 24^{\prime}-2^{\circ} 38^{\prime}$ de longitude Estet couvre plus de $9000 \mathrm{Km}^{2}$. Son régime hydrologique est intimement lié à celui du fleuve Ouémé qui, est lui-même tributaire du climat soudanien (Nord-Bénin). La période de basses eaux est de 8 mois (novembre à juin) tandis que celle des hautes eaux s'étale sur 4 mois (juillet à octobre). Elle communique en plusieurs endroitsavec le complexe lagunaire lac Nokoué lagune de Porto-Novo dans sa partie sud (Balarin, 1984; Chikou, 2006). Avec ses vastes plaines d'inondation, la BVO favorise une importante colonisation par les poissons (Lalèyè et al., 2007). La pêche est une activité séculaire effectuée par les populations autochtones (Toffin, Wémé et Goun) le long du fleuve dans tout le secteur d'étude. Cette activité reste artisanale avec l'utilisation de divers engins et techniques de pêche (Sohou et al., 2009)

Echantillonnage des zones de pêcheries. Les zones de pêcherie - portions de fleuve intensément et habituellement exploitées par les populations pour la pêche - ont été choisies en se basant sur leur proximité avecles agglomérations des pêcheurs, leur accessibilité et l'existence d'un débarcadère d'au moins 10 pirogues,preuve de l'effectivité de l'activité de pêche. De ces critères, 16 zones de pêcheriesont été identifiée (Fig. 1). Dans le souci d'avoir une emprunte spatiale d'échantillonnage représentative et de ne pas empiéter sur les limites des localités, une parcelle de collecte de $500 \mathrm{~m}$ de rayon a été prospectée par zone de pêcherie pour servir de lieux de collecte des données utilisées dans la présente étude.

Collecte de données. Les données de pêche ont été collectées pendant une période de 13 mois (mai 2015 - mai 2016) dans les 16 zones de pêcheries identifiées. La période de collecte a été subdivisée en période de hautes et de basses eaux pour constituer deux blocs dans lesquels les mois de collecte constituent les traitements. Au niveau de chacune de ces zones, deux (2) jours de collecte ont été choisis de façon aléatoire pour chaque traitement. Pour rendre le choix de ces jours stochastique, deux urnes ont été constituées. La ${ }^{1}$ ìe urne contient les initiaux des zones de pêcherie et la $2^{\text {ème }}$ urne renferme les jours d'observation noté. Un tirage aléatoire sans remise a été opéré dans chaque urne afin d'associer à chaque zone tirée les deux jours indépendants de prospection. Le processus est répété chaque mois afin de tenir compte de la variabilité spatio-temporelle des variables à mesurer.

Ces donnéesde pêche collectées sont relatives aux engins et techniques de pêche et, aux espèces de poissons capturées par les pêcheurs. Par jours, tous les types d'engins et techniques de pêche utilisés et leurs caractéristiques sont renseignés sur une fiche de collecte pour chaque zone de pêcherie. Le nombre de pêcheurs exploitant chaque type d'engins et techniques est également noté. Ce procédé a permis d'identifier huit (8) engins et techniques de pêche et 180 pêcheurs au cours de la période d'étude.Concernant les espèces de poissons, les individus présents dans les captures (1918 poissons appartenant à 57 espèces) ont été identifiés (clé d'identification de Paugy et al. (2004), comptés, pesés et mesurés tout au long de la durée de la collecte. Chacun de ces spécimens a été disséqué de l'anus vers la mâchoire inférieure à l'aide d'une paire de ciseaux et les gonades ont été microscopiquement examinées (Ahouansou Montcho et al., 2016). Ces différentes manipulations ont permis d'obtenir 


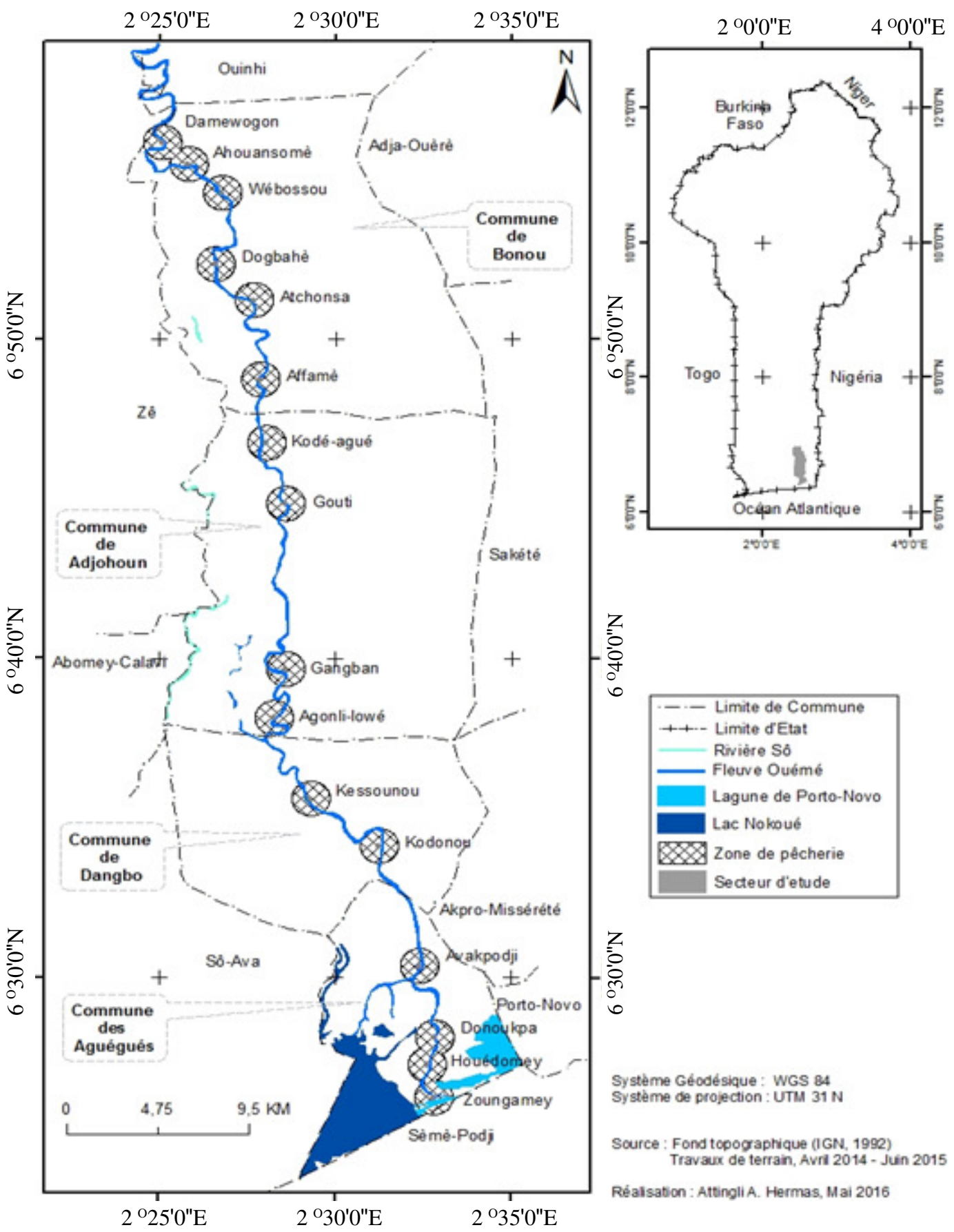

Figure 1. Localisation des zones de pêcheries prospectées dans la BVO. 
: (i) la longueur totale à l'aide d'un ichtyomètre gradué, (ii) le stade de maturité (BrownPeterson et al., 2011) et (iii) la présence et l'abondance des espèces par zone de pêcherie et par commune.

\section{Traitement et analyse des données}

Taux d'utilisation des engin set techniques de pêche dans la basse vallée de l'Ouémé. Le taux d'utilisation $\left(\mathrm{T}_{\mathrm{u}}\right)$ d'un engin ou technique de pêche exprime le taux moyen de réponse relative àl'utilisationd'un engin ou technique par les pêcheurs sur l'ensemble des zones de pêcherie prospectées durant toute la période de l'étude. Il a été déterminé pour chaque engin ou technique de pêche à partir de la formule déduite des données d'enquête :

$T_{u}(e)=\frac{1}{k} \sum_{i=1}^{k}\left(\frac{n_{e}(i)}{n}\right)$

$n_{e^{(i)}}$ : nombre de pêcheurs de la zone de pêcherie (i) ayant utilisé l'engin ou la technique de pêche (e) au cours de la période d'étude ; n: nombre total de pêcheurs ayant utilisé l'engin ou la technique de pêche (e) sur l'ensemble des zones de pêcherie; $\mathrm{k}$ : nombre total de zones de pêcherie prospectées.

Les taux calculés ont subi une transformation racine carrée puis comparés entre engins à partir d'une analyse de variance à un facteur. Une structuration de ces taux a ensuite été opérée afin de regrouper les engins ou techniques de pêche au seuil de $5 \%$. Pour ce faire, le test de structuration de Scheffe (1959) a été utilisé. Le logiciel SPSS 21.0 a été mis à contribution dans l'analyse des données.

Détermination de l'indice de nuisance des engins et techniques de pêche dans la basse vallée de l'Ouémé. L'indice de nuisance (IN) exprime la capacité d'un engin ou d'une technique de pêche à impacter efficacement les peuplements de poissons dans le milieu.
Cet indice estune combinaison linéaire des valeurs de présence des individus des différentes captures par stades de développement $\left(\mathrm{S}_{\mathrm{e}}\right)$, par classes de longueur totale $\left(\mathrm{L}_{\mathrm{e}}\right)$ et par engins et techniques de pêche utilisés $\left(R_{e}\right)$. L'indice de nuisance a été déterminé pour les différents engins et techniques de pêche identifiés sur l'ensemble du secteur d'étude et classés par force croissante de nuisance.

Les cinq (5) étapes suivantes ont été nécessaires pour calculer l'indice de nuisance des engins et techniques de pêche sur le stock disponible.

Etape 0. Détermination des classes de longueur totale des espèces. Pour déterminer les classes de longueur totale à utiliser, on s'est basé sur les longueurs auxquelles 25,50 et $75 \%$ les poissons ont été capturés. La courbe sigmoïde a été utilisée pour la détermination de ces différentes longueurs. Ainsi, la taille de la première capture a été assimilée à la longueur totale (L50c) à laquelle $50 \%$ des poissons seraient capturés par un engin ou technique de pêche (Plisnier, 1990; Ahouansou Montcho et al., 2016). En portant sur un graphique la proportion des poissons capturés en fonction de leur longueur totale on a obtenu la courbe sigmoïde de la Figure 2. L'équation de cette courbe se présente comme suit :

$$
p=\frac{e^{(-5,022+0,43 * L t)}}{\left(1+e^{(-5,022+0,43 * L t)}\right)}
$$

$p$ : pourcentages cumulés de poissons capturés ; $L t$ : longueur totale en $\mathrm{cm}$.

Les longueurs aux quelles $25 \%\left(\mathrm{Q}_{1}\right), 50 \%$ $\left(\mathrm{Q}_{2}\right)$ et $75 \%\left(\mathrm{Q}_{3}\right)$ des poisons sont mature ont été respectivement de 9,15, 10,75 et 12,63 $\mathrm{cm}$. Ces longueurs ont été déduites de la courbe après une linéarisation (Dagnelie, 1973) et une transformation logarithmique de 


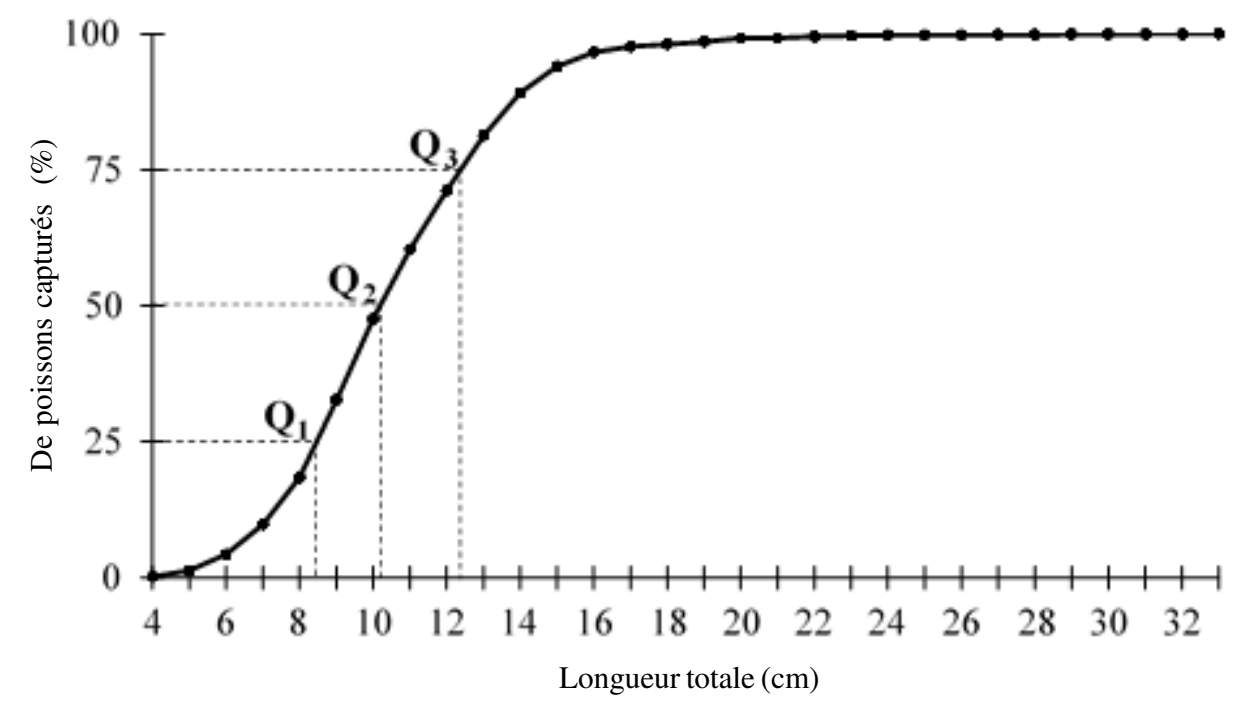

Figure 2. Courbe sigmoïde d'évolution des individus capturés en fonction de la longueur totale.

l'équation de la courbe sigmoïde (Ahouansou Montcho et al., 2016) aboutissant à :

$$
\operatorname{Ln}\left[\frac{p}{(1-p)}\right]=-5,022+0,43 * L t
$$

$$
\left(R^{2}=92,76 \% ; r=0,998 ; p<2,210^{-16}\right)
$$

Les valeurs minimale $(4,2 \mathrm{~cm})$ et maximale $(33,5 \mathrm{~cm})$ de l'échantillon constitué par les longueurs mesurées ont été associées aux longueurs précédemment calculées pour construire une échelle d'appréciation de ces dernières. Ainsi, on admet que les poissons de petite taille ont une longueur variant de 4,2 et $9,15 \mathrm{~cm}$, ceux de taille moyenne ont une longueur oscillant entre 9,15 et $12,63 \mathrm{~cm}$ tandis que les poissons de grande taille ont une longueur comprise entre 12,63 et $33,5 \mathrm{~cm}$.

Etape 1. Détermination de la $S_{e} \cdot$ La $S_{e}$ exprime la valeur de présence des espèces capturées en fonction des stades de maturité (Tableau 2) au niveau de chaque engin ou technique de pêche sur l'ensemble du secteur d'étude. Elle a été calculée à partir de la formule:

$$
S_{e}=\frac{f_{s}(e)}{\sum_{e=1}^{k} f_{s}(e)} \text { avec } f_{s}(e)=\frac{n_{s}(e)}{n}
$$

$f_{s}(e)$ : présence des espèces capturées par l'engin ou technique de pêche $(e) ; n_{s}(e)$ : nombre des $s$ stades de maturitéauxquels les individusont été capturées par l'engin oula technique de pêches $(e) ; n$ : nombre total de stades de maturité identifiés ; $k$ : nombre d'engins et techniques de pêche sélectionnés.

Etape 2. Détermination de la $L_{e}$ La $L_{e}$ exprimela valeur de présence des espèces capturéesen fonction des classes de longueur totale par engins ou technique de pêche sur l'ensemble du secteur d'étude. Elle a été déterminée à partir de la formule :

$$
L_{\epsilon}=\frac{f_{l}(\theta)}{\sum_{\epsilon=1}^{k} f_{l}(\theta)} \text { avec } f_{l}(\theta)=\frac{\omega_{l}(\theta)}{\omega}
$$


TABLEAU 1. Echelle de maturité des gonades de poissons

\begin{tabular}{|c|c|c|}
\hline Stades & Femelles & Mâles \\
\hline I & $\begin{array}{l}\text { Ovaires non différenciés avec des filaments } \\
\text { blanchâtres à peine transparents }\end{array}$ & $\begin{array}{l}\text { Testicules non différenciés avec des filaments } \\
\text { blanchâtres à peine transparents }\end{array}$ \\
\hline II & $\begin{array}{l}\text { Ovaires différenciés, ovocytes très petits } \\
\text { mais non libres }\end{array}$ & $\begin{array}{l}\text { Testicules déjà développés mais pas de sperme visible } \\
\text { après incision }\end{array}$ \\
\hline III & $\begin{array}{l}\text { Ovaires non différenciés, ovocytes gros et } \\
\text { plus ou moins libres mais non expulsables par } \\
\text { pression manuelle sur l'abdomen }\end{array}$ & $\begin{array}{l}\text { Sperme visible après incision des testicules mais non } \\
\text { expulsables par pression manuelle sur l'abdomen }\end{array}$ \\
\hline IV & Ovules expulsables à la pression manuelle & Sperme expulsable à la pression manuelle \\
\hline $\mathrm{V}$ & Ovaires vides, à l'état du stade II & Testicules vides, à l'état du stade II \\
\hline
\end{tabular}

Source: Brown-Peterson et al. (2011)

$f_{l(e)}$ : présence des espèces capturées par l'engin ou la technique $(e) ; \omega_{1(e)}$ : nombre des $l$ classes de longueur totale auxquelles les espèces ont été capturées par l'engins ou la technique de pêches $(e)$; w: nombre total de classes de longueur totale identifiées ; $k$ : nombre d'engins et techniques de pêche sélectionnés.

Etape 3. Détermination de la $\boldsymbol{R}_{\boldsymbol{e}}$. La $R_{e}$ exprime la valeur relative du nombred'espèce capturée par engin et technique de pêche sur l'ensemble du secteur d'étude. Elle a été déterminée à partir de la formule :

$$
R_{\epsilon}=\frac{f_{r}(\theta)}{\sum_{\epsilon=1}^{k} f_{\gamma}(\theta)} \text { avec } f_{r(\theta)}=\frac{\theta_{r(\theta)}}{\theta}
$$

$f_{r(e)}:$ présence relative des espèces capturés par l'engin ou technique de pêches $(e) ; \theta_{r(e)}$ : nombre des $r$ individus capturés par l'engin

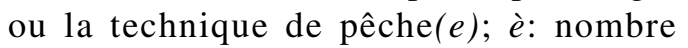
d'individusidentifiés sur l'ensemble du secteur d'étude; $k$ : nombre d'engins et techniques de pêche sélectionnés.

Etape 4. Détermination de l'indice de nuisance $(I N)$. Le $I N$ est l'importance relative de la composition des captures par engins et techniques de pêches utilisés dans le secteur d'étude. Cet indice a été calculé pour chaque engin ou technique de pêche et a pour formule:

$$
I N=\frac{1}{3}\left(S_{e}+L_{e}+R_{e}\right)
$$

L'indice varie entre 0 et 1 . La force de nuisance d'un engin ou technique de pêche sur le stock disponible est faible lorsque l'indice tend vers 0 . Mais, cette force est élevée lorsque l'indice tend vers 1 .

Pour apprécier les niveaux de nuisance des engins et techniques de pêche, les valeurs de l'indice de nuisance obtenues ont ététriées par ordre croissant et découpées par la méthode des quartiles. A l'issus de cette analyse, une échelle d'appréciation a été créée : Faible $\left(I_{\text {inf }-} Q_{1}\right)$, Moyenne $\left(Q_{1-} Q_{3}\right)$ et Forte $\left(Q_{3-} I N_{\text {sup }}\right)$. Avec $Q_{1}$ et $Q_{3}: 1^{\text {er }}$ et $3^{\text {ème }}$ quartile de la série considérée, ${ }^{I N}{ }_{\text {inf }}$ : la plus petite valeur de l'indice de nuisance et ${ }^{I N_{\text {sup }}}$ : la plus grande valeur de l'indice de nuisance de la série. 
TABLEAU 2. Engins et techniques de pêche fréquemment utilisés dans le secteur d'étude

Engin et techniques de pêche Caractéristiques

Ligne« alonouhou »

Nasse

Barils « Gbadja »

Palangre

Epuisette géante « Dobah»

Filet épervier

Filet maillant

Parcs à branchages« Acadja »

Source : Enquête de terrain (Attingli, 2014)
Canne à pêche muni d'un hameçon appâté. Il est surtout exploité par les enfants et les femmes sur les berges

Pièges fabriqués avec des nervures de palmes ou de bambou fendues, disposé le longd'une palissade en branche de palme pour capturer les poissons.La charpente est entourée de filet en nylon multifilament de $1,2 \mathrm{~cm}$ de maille nœud à nœud dans lequel sont pratiqué latéralement 2 à 4 ouvertures

Baril de a75 cm de long, percé d'un trou (rayon $15 \mathrm{~cm}$ ) recouvert d'un grillage (maille $50 \mathrm{~mm}$ ) appâté d'unChrysichthys. Le son émis par leChrysichthys depuis le baril attire d'autres qui se font piéger à leur tour. A l'aide d'une corde $(5$ à $8 \mathrm{~m})$ préalablement fixée à un piquet qui retient le baril, deux pêcheurs peuvent remonter 3 jours après le «Gbadja » pour en extraire le contenu

Ligne principale de $80 \mathrm{~cm}$ environ à laquelle est attachée des avançons.Les lignes sont faites d'un fil principale le long duquel sont attachés à des écartements presque régulier de nombreux avançons terminés par des hameçons appâtées

Le « Dobah » est une grande épuisette montée sur un cadre de bois en cercle attaché à un long manche. Il estmuni de filet de petites mailles (5à8mm entre les nœuds) et/oude grandes mailles (20 à $30 \mathrm{~mm}$ entre les nœuds)

Les éperviers sont des engins de formes coniques évasées. Au sommet du cône est attachée une corde (5à10m). Ils sont montés surplace en fil nylon multi-filament. Les mailles sont très petites (10 et 30mm).Mais,on en rencontre aussi de grandes mailles (15-25 mm à Houando)

Ils sont constitués de nappe de forme rectangulaire.Seuls les ralingues inférieures sont munies de plomb, ou de bourrelets d'argile cuits. Les mailles sont variables $(5$ et $30 \mathrm{~mm}$ ) et s'étalent sur 35 à $40 \mathrm{~m}$ de long avec une hauteur de chute de 1 à $2 \mathrm{~m}$

Il s'agit d'un dispositif permanent fait de branchage et de végétation flottante à dominance de Pistias stratiotes, Echlornia crassipe et pomea aquatica. Le dispositif attire les poissons et assure leur alimentation (micro-organismes) 
Détermination du niveau d'impact des engins et techniques de pêche sur le stock disponible dans la basse vallée de l'Ouémé. L'indice d'impact (II) exprime la force pondérale des effets de nuisance des engins et techniques de pêche sur la population des espèces de poissons dans la basse vallée de l'Ouémé. Cet indice estune combinaison linéaire de la valeur des occurrences des espèces des différentes captures par stades de développement $\left(I S_{z}\right)$, par classes de longueur totale $\left(I L_{z}\right)$ et par engins et techniques de pêche utilisés $\left(I R_{z}\right)$. Il a été calculépour les différentes zones de pêche et pour lesCommunes du secteur d'étude puis classé par niveau d'impact.

Les quatre (4) étapes suivantes ont été nécessaires pour déterminer l'indice d'impact des engins de pêche dans la Basse Vallée de l'Ouémé.

Etape 1: Détermination de I'IS. L'IS exprime l'occurrence relative des espèces capturées par les engins et techniques de pêche utilisés dans une zone de pêcherie en fonctiondes stades de développement des espèces identifiées. Elle a été calculée à partir de la formule :

$$
\begin{aligned}
& I S_{z}=\sum_{e=1}^{k}\left(\sum_{i=1}^{s} S_{z}(i, e)\right)_{e} \text { avec } \\
& S_{z}(i, e)=\frac{\alpha_{z}(i, e)}{n}
\end{aligned}
$$

$\alpha_{z(i, e)}$ : occurrencedes espèces capturées pour le $i^{\text {eme }}$ stade de développement par un engin ou technique de pêche $(e)$ dans une zone de pêcherie $(z) ; S_{z(i, e)}$ : proportion d'espèces capturées pour le $i^{\text {eme }}$ stade de maturité par un engin ou technique de pêche $(e)$ dans une zone de pêcherie $(z) ; n$ : occurrence totaled'espèces capturées sur l'ensemble des zones de pêcherie; $s$ : nombre de stades de développement identifiés ; $k$ : nombre d'engins et technique de pêche utilisés dans la zone de pêcherie $(z)$.

Etape 2. Détermination de l' $I L_{z}$. L'IL exprime l'occurrence relative des espècescapturées par les engins et techniques de pêcheutilisés dans une zone de pêcherie en fonction des classes de longueur totale obtenues. Elle a été déterminée à partir de la formule :

$$
\begin{aligned}
& I L_{z}=\sum_{e=1}^{k}\left(\sum_{\delta=1}^{l} L_{z}(\delta, e)\right)_{e} \text { avec } \\
& L_{z}(\delta, e)=\frac{\beta_{z}(\delta, e)}{n}
\end{aligned}
$$

$\beta_{z(\delta, e)}$ : occurrence des espèces capturées pour la $\ddot{a}^{\text {eme }}$ classe de longueur totale par un engin ou technique de pêche $(e)$ dans une zone de pêcherie $(z) ; L_{z(\delta, e)}$ : proportion d'espèces capturées pour la $\ddot{a}^{\text {ème }}$ classe de longueur totale par un engin ou technique de pêche $(e)$ dans une zone de pêcherie $(z) ; n$ : occurrence totale d'espèces capturées sur l'ensemble des zones de pêcherie ; $l:$ nombre de classes de longueur totale identifiées ; $k$ : nombre d'engins et technique de pêche utilisés dans la zone de pêcherie $(z)$.

Etape 3. Détermination de l'IR. L'IR exprime l'occurrence relative des espèces par engins et technique de pêche sur l'ensemble des zones de pêcherie au cours de la période d'étude. Elle a été calculée à partir de la formule:

$$
I R_{z}=\sum_{i=1}^{k} R_{z j}(\theta) \text { avec } \quad R_{z j}(\theta)=\frac{r_{z j}(\theta)}{n}
$$

$r_{z j(e)}:$ occurence des $j$ espèces capturées par l'engin ou la technique de pêche $(e)$ dans la zone de pêcherie $(z) ; R_{z j(e)}$ : proportion des $j$ espèces capturées par l'engin ou la technique de pêche $(e)$ dans la zone de pêcherie $(z) ; n$ : occurrence totale des espèces capturées sur l'ensemble des zones de pêcherie ; $k$ : nombre 
d'engins et technique de pêche utilisés dans la zone de pêcherie $(z)$.

Etape 4. Détermination de l'indice d'impact (II). L'II est le poids relatif de l'impact réel des engins et techniques de pêche sur la population des espèces dans les zones de pêcherie sur l'ensemble du secteur d'étude. L'indice d'impact a été calculé par la formule:

$$
I I_{z}=\frac{1}{\lambda}\left(I S_{z}+I L_{z}+I R_{Z}\right)
$$

$\lambda$ : nombre de paramètres présents dans la formule. Il est égal à 3 dans la présente formule.

Pour connaître la valeur de l'indice d'impact des engins et techniques de pêche dans les Communes de la BVO, la formule ciaprès a été définie :

$$
I I_{c}=\frac{1}{\pi} \sum_{\mathrm{z}=1}^{\pi} I I_{z}
$$

$\partial$ : nombre de zones de pêcherie présentes dans une Commune $c$.

L'indice d'impact des engins et techniques de pêche varie entre 0 et 1 . L'impact des engins et techniques de pêche sur le stock disponible est faible lorsque l'indice tend vers 0 . Il est élevé dans le secteur considéré lorsque sa valeur tend vers 1 .

Pour définir les niveaux d'impact des engins et techniques de pêche, les valeurs de l'indice d'impact obtenues ont ététriées par ordre croissant et découpées par la méthode des quartiles. A l'issus de cette analyse, une échelle d'appréciation a été créée : Faible $\left(I I_{\text {inf- }} Q_{1}\right)$, Moyenne et Forte (. Avec: $1^{\text {er }}$ et $3^{\text {ème }}$ quartile de la série considérée, : la plus petite valeur de l'indice d'impact et : la plus grande valeur du même indice.

Afin d'avoir une cartographie del'impact des engins et techniques de pêche dans le secteur d'étude, une spatialisation des valeurs d'impact a été faite par interpolation linéaire en utilisant l'interpolateur de distance inverse pondérée (IDW: Inverse Distance Weighted). La valeur choisie pour le paramètre de puissance est 2. La résolution de la grille a été de $50 \mathrm{~m}$. L'extension spatial analyst et le module interpolation du logiciel ArGIS 9.3 ont été utilisés pour l'analyse.

Détermination de l'abondance relative des espèces capturées dans la basse vallée de l'Ouémé. L'abondance relative des espèces capturées $(A R)$ exprime l'intégration des abondances des espèces par captures et par moissur l'ensemble des zones de pêcheriesau cours de la période d'étude.

Les trois (3) étapes suivantes ont été nécessaires pour calculer l'abondance relative des espèces capturées par zone de pêcherie et par Commune de la Basse Vallée de l'Ouémé.

Etape 1. Détermination de l'abondance spécifique par capture $\left(A C_{j}\right)$. L'AC exprime le nombre moyen d'individus d'une espèce quelconque par capture. Elle est déterminée à partir de la formule:

$$
A C_{j}=\frac{1}{\gamma} \sum_{i=1}^{\gamma} A_{j(i)}
$$

$A_{j(i)}:$ nombre total d'individus de l'espèce $j$ au $i^{e m e}$ capture ; : nombre total de captures.

Etape 2. Détermination de l'abondance spécifique mensuelle $\left(\boldsymbol{A} \boldsymbol{M}_{m}\right)$. L'AM exprime l'abondance mensuelle d'une espèce quelconque sur l'ensemble du secteur d'étude. Elle est déterminée à partir de la formule:

$$
A M_{m}=\underline{1} \sum_{j=1}^{\tau} A C_{(j)}
$$

$\tau$ : nombre total espèce capturée dans le $m^{\text {ême }}$ mois de prospection. 
Etape 3. Détermination de l'abondance relative $(\boldsymbol{A R})$. L'abondance relative des espèces capturées par zone de pêcherie $\left(A R_{z}\right)$ a été déterminée par la formule :

$$
A R_{z}=\frac{1}{\vartheta} \sum_{\mathrm{m}=1}^{\vartheta} A M_{(m)}
$$

$\vartheta$ : nombre total de mois de collecte.

L'abondance relative des espèces capturées par commune $\left(A R_{c}\right)$ a été déterminée par la formule:

$$
A R_{c}=\frac{1}{\rho} \sum_{\mathrm{z}=1}^{\rho} A R_{\mathrm{z}}
$$

$p:$ nombre de zones de pêcherie présentes dans une commune $c$.

Afin de classer les zones de pêcheries et les Communesdu secteur d'étude suivant les abondances relatives des espèces capturées, les valeurs d'abondance relative obtenues ont été triées par ordre croissant et découpées par la méthode des quartiles. A l'issus de cette analyse, une échelle d'appréciation a été créée $:$ Faible, Moyenne et Forte (. Avec : $1^{\text {er }}$ et $3^{\text {ème }}$ quartile de la série considérée, : la plus petite valeur d'abondance relative et $:$ la plus grande valeur l'abondance relative de la série.

Une cartographie de l'abondance relative des espèces capturées dans la BVO a été faite par interpolation linéaire en utilisant l'interpolateur de distance inverse pondérée (IDW : Inverse Distance Weighted). La valeur choisie pour le paramètre de puissance est 2 . La résolution de la grille a été de $50 \mathrm{~m}$. L'extension spatial analyst et le module interpolation du logiciel ArGIS 9.3 ont été utilisés pour l'analyse.

Relation impact des engins et techniques de pêches et abondance relative des espèces dans la Basse Vallée de l'Ouémé. L'analyse de la relation entre l'abondance relative et l'impact des engins et techniques de pêche a été faite grâce au coefficient de corrélation de Pearson puis testée au seuil de $5 \%$. Lorsque la valeur du coefficient est forte et significative, une analyse de régression linéaire simple a été opérée pour essayer de prédire l'abondance relative (variable à expliquer) à partir des valeurs de l'indice d'impact des engins et technique de pêche (variable explicative). Le modèle de régression utilisé est de la forme:

$$
\operatorname{In}(A R)=a+b^{*} \operatorname{In}(I I)+\varepsilon
$$

$\mathrm{a}$ : ordonnée à l'origine; $\mathrm{b}$ : pente ; $\mathrm{AR}$ : abondance relative; II : valeur de l'indice d'impact ; å : erreur

La significativité global du modèle et celle des coefficients de régression ont été respectivement testées à partir d'une analyse de variance à un facteur fixe (abondance relative) et le test $t$ de Student au seuil de $5 \%$. Les conditions de normalité, d'homogénéité, d'indépendance et de conformité des résidus de régression ont été également testées au seuil de $5 \%$. L'erreur standard résiduelle (ESR) ainsi que le facteur correctif (FC) relatif à la transformation logarithmique ont été calculés. Le facteur de correction a pour formule :

$$
F C=\operatorname{Exp}\left(E S R^{2} / 2\right)
$$

Le logiciel R 3.3.1a été utilisé pour les différentes analyses.

\section{RÉSULTATS}

Description des engins et techniques de pêche dans la Basse Vallée de l'Ouémé. Les huit (8) engins et techniques de pêche fréquemment utilisés dans la basse vallée de l'Ouémé sont : lignes "alonouhou », palangres, filets maillants, épuisettes géantes «Dobah », filets éperviers, nasses, parcs à poisson « acadja » et barils « Gbadja » (Tableau 2). 
Taux d'utilisation des engins et techniques $7 ; \mathrm{P}=0,000)$. Les engins et techniques les de pêche dans la Basse Vallée de l'Ouémé. plus utilisés sont les parcs à branchage La Figure 3 présente les taux d'utilisation des «Acadja » et les Filets maillants suivis des engins et techniques de pêche dans la Basse barils «Gbadja » et les Nasses. Les épuisettes Vallée de l'Ouémé.

L'analyse de cette figure montre que dans l'ensemble, le taux moyen d'utilisation des engins et technique de pêche dans la basse Vallée de l'Ouémé varie de $0,009( \pm 0,003)$ à $0,502( \pm 0,215)$. Ce taux est significativement différent entre engins et techniques de pêche sur l'ensemble des zones de pêcherie et leur périphérie au seuil de $5 \%(\mathrm{~F}=29,88$; ddl $=$ géantes «Dobah», Filets éperviers et Palangres ne sont que moyennement utilisés tandis que l'utilisation des Lignes a été rarement observée dans le secteur d'étude.

Indice de nuisance des engins et techniques de pêche (IN). Le Tableau 3 présente l'indice de nuisance des 8 engins et techniques de pêche recensés dans la Basse Vallée de

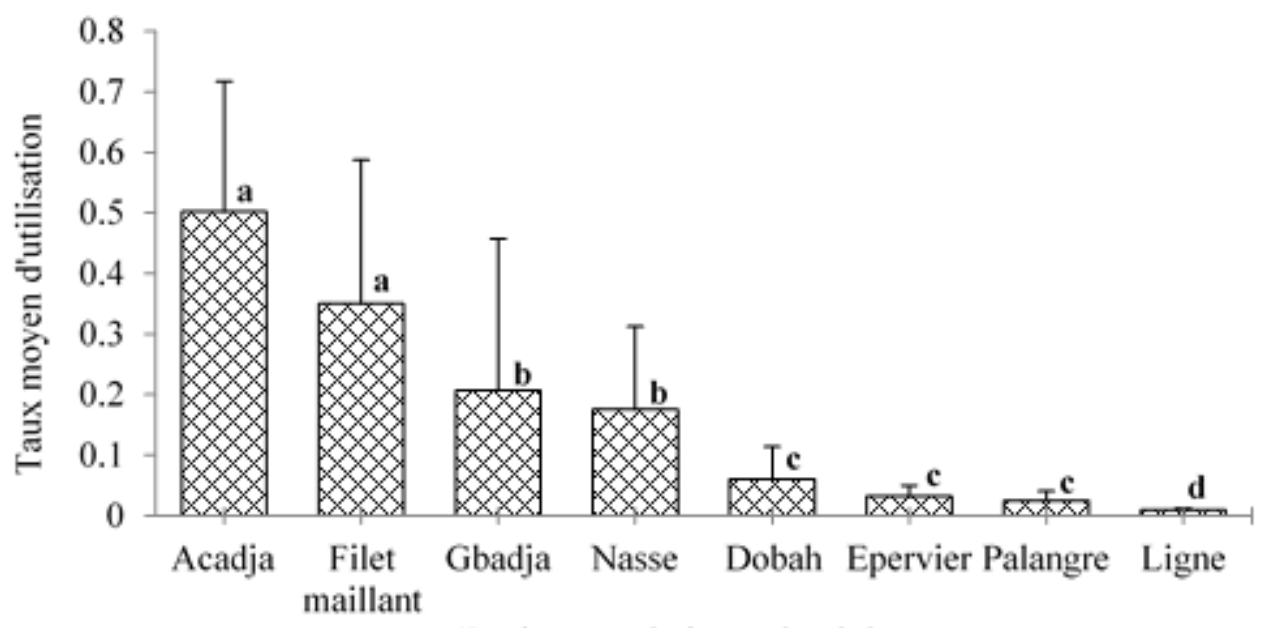

Engins et techniques de pêche

Les barres accompagnées de la même lettre présentent des taux moyens qui ne sont pas significative au seuil de $5 \%$ (test de structuration de Scheffe au seuil de $5 \%$ )

Figure 3. Taux d'utilisation des engins et techniques de pêche dans la Basse Vallée de l'Ouémé.

TABLEAU 3. Indice de nuisance (IN) des engins et techniques de pêchedans le secteur d'étude

\begin{tabular}{lll}
\hline Engins et techniques de pêche & IN & Echelle d'appréciation \\
\hline Ligne & 0,052 & Faible \\
« Gbadja » & 0,076 & \\
Palangre & 0,099 & Moyenne \\
«Dobah » & 0,115 & \\
Epervier & 0,117 & \\
Nasse & 0,122 & Forte \\
«Acadja » & 0,209 & \\
Filet maillant & 0,209 &
\end{tabular}


l'Ouémé.De l'analyse du Tableau 3, il ressort que l'indice de nuisance varie d'un engin ou technique de pêche à l'autre. Les engins tels que leslignes et "Gbadja" présentent les faibles niveaux de nuisances sur le stock $(0,052<$ IN $<0,099)$ comparativement auxPalangre, "Dobah", Epervieret Nasses dont l'indice de nuisance est moyen $(0,099<\mathrm{IN}<0,209)$. Les parcs à branchage "Acadja" et les filets maillants sont les engins et techniques de pêche les plus nuisible au stock dans la BVO (IN > 0,209 ).

Impact des engins et techniques de pêche sur le stock disponible dans la Basse Vallée de l'Ouémé

Occurrence des espèces capturées par stades de maturité. La Figure 4 présente l'occurrence relative des espèces capturées par engins et techniques de pêche et par stades de maturitédans la BVO. L'analyse de cette figure révèle que chaque engin ou technique de pêche capture, dans des proportions variables, des espèces d'au moins deux stades de maturité différents. Dans le sens décroissant du nombre de stade impactés, on note que les « Acadja »,
Filets maillants, Eperviers et les Nasses prennent les espèces de tous les cinq (5) stades de maturité(I, II, III, IV et V) identifiés tandis que les «Dobah» et Palangres ne capturent que des espèces de quatre (4) stades de maturité (I, II, III et IV) et, les Gbadja et Lignes respectivement les espèces de trois (3) stades de maturité (I, II, III) et de deux (2) stades de maturité (I, II). Les espèces des quatre (4) premiers stades de maturité sont plus prépondérantes dans tous les engins et techniques dans lesquels elles ont été prises. Les espèces de stade $V$ ne sont majoritairement capturées que par les Filets éperviers et les Nasses.

\section{Occurrence des espèces par classes de longueur totale. La Figure 5} illustrel'occurrence relative des espèces capturées par engins et techniques de pêche par classes de longueur totale dans la BVO. Chaque engin ou technique de pêche capture des espèces d'au moins deux (2) classes de longueurs totales. La proportion des espèces varie significativement par classe de longueur totale au sein de chaque engin ou technique de pêche. Les «Acadja », Filets maillants, Filets

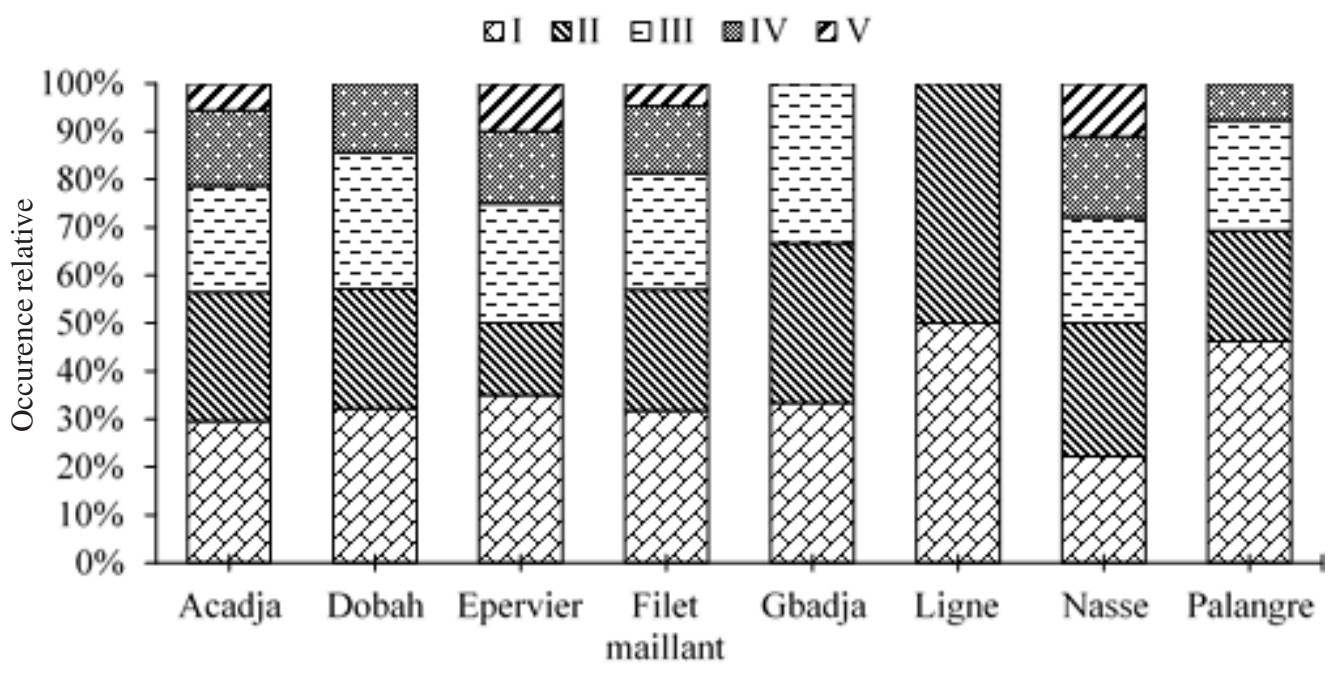

Engins et techniques de pêche

Figure 4. Occurrence relative des espèces capturées par engins et techniques de pêche par stades de maturité dans la BVO. 
éperviers, «Dobah », « Gbadja », Nasses et Palangres capturent les espèces des trois classes de longueur totale alors que les Lignes n'en prennent que celles des deux dernières classes de longueur totale. Les espèces des deux premières classes de longueur totale sont prépondérantes dans les captures de tous les engins et technique de pêche.
Occurrence des espèces par engins et techniques de pêche. La Figure 6 présente la richesse spécifique relative des captures par engins et techniques de pêche dans la BVO. Elle montre que la richesse spécifiquerelative des captures varie suivant les engins et techniques de pêches. Les Filets maillants et «Acadja » présentent les richesses spécifiques

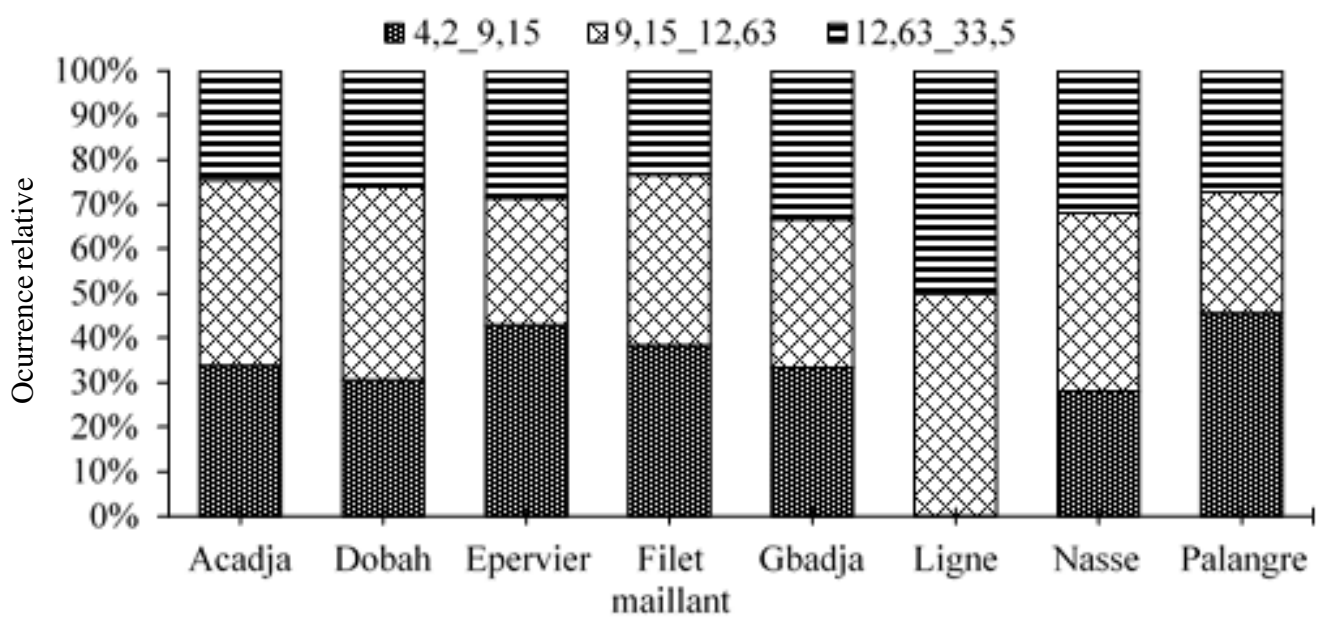

Engins et techniques de pêche

Figure 5. Occurrence relative des espèces capturées par engins et techniques de pêche par classes de longueur totale dans la BVO.

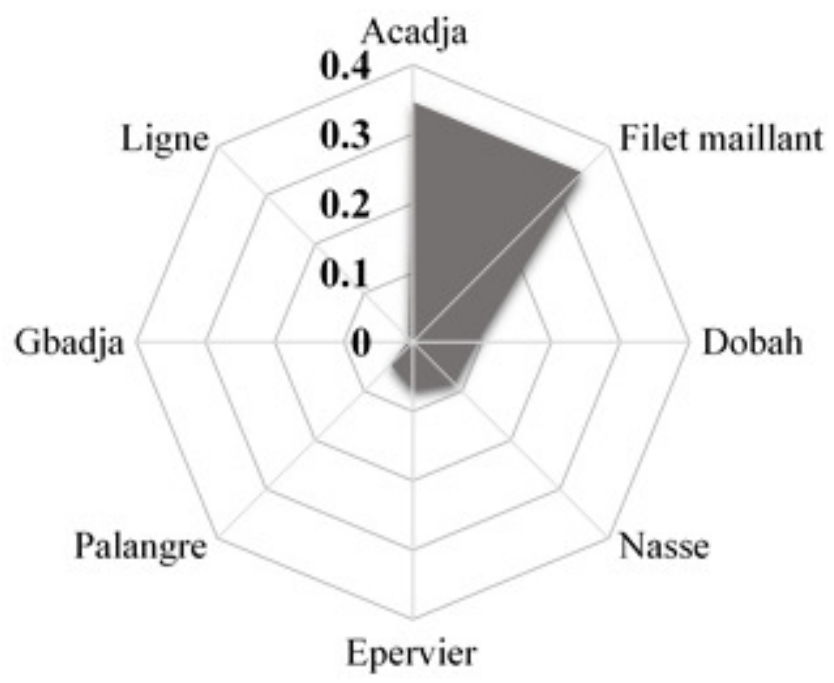

Figure 6. Occurrencerelative des espèces par engins et techniques de pêche dans la BVO. 
les plus élevées. Les autres engins et techniques de pêche ne sont caractérisés que par de faibles richesses spécifiques.

Indice d'impact des engins et techniques de pêche sur le stock disponible dans la BVO. De l'analyse du Tableau 4, il ressort qu'il existe globalement un gradient croissant d'impact des engins et techniques de pêche de l'amont vers l'aval de la BVO. Ce qui permet de grouper les zones de pêcherie en trois blocs. Le premier bloc est majoritairement composé des zones de pêcherie situées en amont du secteur d'étude dans la Commune de Bonou et de Adjohoun (Dogbahè, Ahouansomé,Kodéagué et Gouti). Ces zones sont caractérisées par de faible emprise des engins et techniques de pêche $(0,007<$ II $<0,015)$. Le deuxième bloc est celui des zones de pêcherie dans lesquelles l'emprise des engins et techniques de pêche est moyenne sur le stock disponible $(0,015<\mathrm{II}<0,1)$. Il s'agit des zones de pêcherie de Wébossou,Affamè, Damewogon et d'Atchonsa (en amont), les zones de pêcherie de Gangban, Kessounou et Kodonou (au centre) et celle de Houédomey (en aval).
Comparées aux zones des deux premiers blocs, les zones de pêcherie du bloc 3 sont celles situées en aval du secteur d'étude dans la Commune des Aguégués (Avakpodji, Donoukpa et Zoungamey) et singulièrement celle d'Agonlin-lowé dans la Commune de Adjohoun au centre de la BVO. Ces zones de pêcherie sont caractérisées par une forte emprise des engins et techniques de pêche sur les stocks de poissons $(0,1<$ II $<0,146)$.

Du Tableau 5, il ressort que les Communes de Bonou et des Aguégués sont significativement opposées en termes d'impact des engins et techniques de pêche sur le stock disponible. Les faibles valeurs des coefficients de variation pour les indices des Aguégués et Dangbo révèlent une certaine uniformité spatiale de l'impact dans les pêcheries de ces Communes comparativement aux autres Communes où une très grande variation est observée (Fig. 7).

\section{Abondance relative des espèces par capture dans la BVO. L'analyse du Tableau 6 révèle l'existence un gradient croissant d'abondance relative des espèces capturées de l'amont vers}

TABLEAU 4. Indice d'impact (II) des engins de pêche sur le stock disponible par zone de pêcherie

\begin{tabular}{lcc}
\hline Zones de pêcherie & II & Echelle d'appréciation \\
\hline Kodé-agué & 0,007 & Faible \\
Gouti & 0,009 & \\
Dogbahè & 0,013 & \\
Ahouansomè & 0,015 & \\
Wébossou & 0,016 & Moyen \\
Affamè & 0,016 & \\
Kessounou & 0,038 & \\
Damewogon & 0,042 & \\
Gangban & 0,061 & \\
Atchonsa & 0,067 & \\
Kodonou & 0,072 & \\
Houédomey & 0,100 & Fort \\
Agonli-lowé & 0,126 & \\
Avakpodji & 0,134 & \\
Donoukpa & 0,136 & \\
Zoungamey & 0,146 & \\
\hline
\end{tabular}


TABLEAU 5. Indice d'impact (II) des engins et techniques de pêche sur le stock disponible parCommune dans la BVO

\begin{tabular}{lccl}
\hline Commune & \multicolumn{2}{c}{ II } & Echelle d'appréciation \\
\cline { 2 - 3 } & $\mathrm{m}$ & $\mathrm{Cv}(\%)$ & \\
\hline Bonou & 0,028 & 77,08 & Faible \\
Adjohoun & 0,051 & 110,74 & Moyenne \\
Dangbo & 0,055 & 43,86 & \\
Aguégués & 0,129 & 15,57 & Forte \\
\hline
\end{tabular}

$\mathrm{m}$ : Moyenne arithmétique $; \mathrm{cv}$ : coefficient de variation en pourcentage

TABLEAU 6. Abondance relative des espèces par zone de pêcherie dans le secteur d'étude

\begin{tabular}{lll}
\hline Zone de pêcherie & AR & Echelle d'appréciation \\
\hline Gouti & 0,590 & Faible \\
Kodé-ague & 0,824 & \\
Affanmè & 0,977 & \\
Dogbahè & 1,072 & \\
& & \\
Kessounou & 1,212 & Moyenne \\
Kodonou & 1,295 & \\
Atchonsa & 1,324 & \\
Ahouansonmè & 1,541 & \\
Wébossou & 1,553 & \\
Damewogon & 1,673 & \\
Gangban & 2,321 & \\
Donoukpa & 2,387 & \\
& & \\
Avakpodji & 2,482 & Fort \\
Zoungamey & 2,867 & \\
Houédomey & 2,888 & \\
Agonli-Lowé & 3,723 & \\
\hline
\end{tabular}

l'aval de la BVO. Ainsi, trois groupes de zones de pêcherie peuvent être identifiées. Le premier groupe est celui des zones de pêcherie ayant une faible abondance relative en espèces $(0,590$ $<\mathrm{AR}<1,072$ ). Il est principalement constitué des zones de pêcheries situées en amont du secteur d'étude telles que Affanmè et Dogbahé (Commune de Bonou) et, Kodé-agué et Gouti (Commune d'Adjohoun). Quant au deuxième groupe, il renferme les zones de pêcheries (Atchonsa, Ahouansonmè, Wébossou, Damewogon Kodonou, Kessounou, Gangban et Donoukpa) dont les abondances relatives en espèces sont moyennes du point de vue captures $(1,072<\mathrm{AR}<2,387)$. Le dernier groupe est constitué des zones de pêcheries où l'on enregistre les fortes abondances relatives des espèces par captures $(2,372<\mathrm{AR}$ $<3,723)$. Ces zones de pêcheries sont situées en aval de la BVO dans les Communes des Aguégués (Avakpodji, Zoungamey et Houédomey) et d'Adjohoun (Agonlin-lowé).

De l'analyse du Tableau 7, il ressort une distribution globalement croissante de l'abondance relative des captures de l'amont (Commune de Bonou) vers l'avales (Communes des Aguégués). Cependant, une inflexion est notée dans la Commune de Dangbo. Par ailleurs, les faibles variations de l'abondance relative entre les zones de pêcherie des Communes de Dangbo, Aguégués et Bonou révèlent son uniformité spatiale dans ces Communes comparativement à la Commune d'Adjohoun où une très grande variation est observée entre zones de pêcherie (Fig. 8).

Relation engins et techniques de pêches et abondance relative des espèces dans la BVO. Les résultats de l'analyse de corrélation révèle une relation positive forte hautement significative entre l'abondance relative (AR) etla valeur de l'indice d'impact (II) des engins et techniques de pêche (Tableau 8) au seuil de $5 \%\left(\mathrm{r}=0,849 ; \mathrm{P}=3,12^{*} 10^{-5}\right)$. La validation du modèle par le test d'analyse de variance a révélé que le modèle obtenu est globalement 


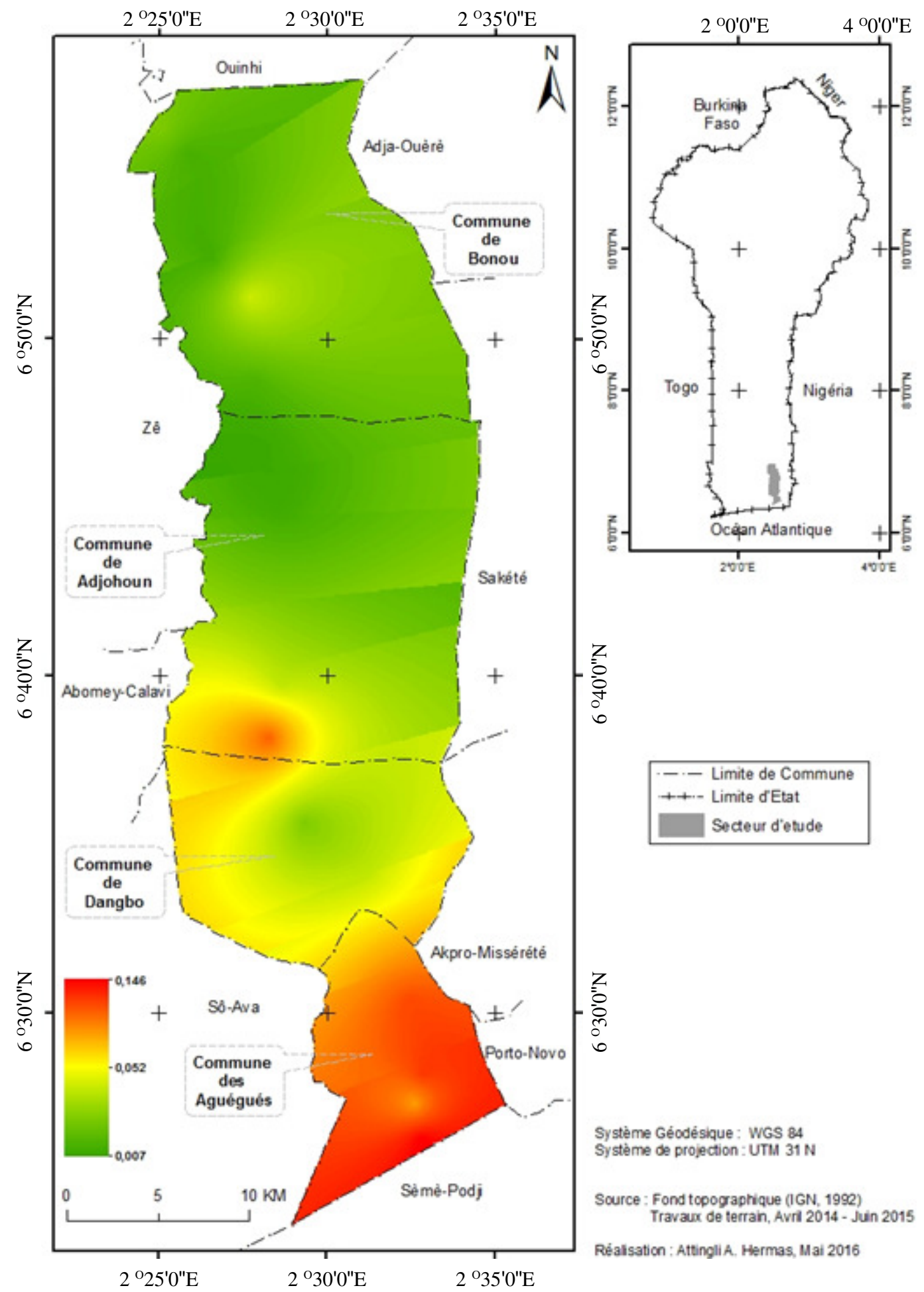

0.148 à 0.100 : secteurs à fort impact des engins et techniques de pêche. 0.090 à 0.056 : secteurs à impact moyen des engins et techniques de pêche. 0.060 à 0.007 : secteurs à faible impact des engins et techniques de pêche

Figure 7. Spatialisation de l'impact des engins de pêche dans la BVO. 
TABLEAU 7. Abondance relative des espèces capturées par Commune dans la Basse Vallée de l'Ouémé

\begin{tabular}{lcrl}
\hline Commune & \multicolumn{2}{c}{ AR } & Echelle d'appréciation \\
\cline { 2 - 3 } & $\mathrm{m}$ & $\mathrm{Cv}(\%)$ & \\
\hline Dangbo & 1,253 & 4,71 & Faible \\
Bonou & 1,357 & 20,83 & Moyenne \\
Adjohoun & 1,864 & 78,15 & Fort \\
Aguégués & 2,656 & 9,75 & \\
\hline
\end{tabular}

$\mathrm{m}$ : Moyenne ; cv : Coefficient de variation en pourcentage

TABLEAU 8. Corrélation entre abondance relative des espèces et différents facteurs du milieu

\begin{tabular}{lll}
\hline Variables & \multicolumn{2}{c}{ Abondance relative } \\
\cline { 2 - 3 } & $\mathrm{r}$ & $\mathrm{p}$ \\
\hline Facteur d'anthropisation du milieu & & \\
II & 0,849 & $<0,0001$ \\
\hline
\end{tabular}

TABLEAU 9. Caractéristiques et test de significativité des coefficients de régression

\begin{tabular}{|c|c|c|c|c|c|}
\hline \multirow[t]{2}{*}{ Coefficients estimés } & \multicolumn{3}{|c|}{ Caractéristiques } & \multicolumn{2}{|c|}{ Test t de Student } \\
\hline & Valeur & $\mathrm{SE}$ & IC $(95 \%)$ & $\mathrm{t}$ & $\mathrm{p}$ \\
\hline$\hat{a}$ & $\begin{array}{l}1,780 \\
0,409\end{array}$ & $\begin{array}{l}0,228 \\
0,067\end{array}$ & $\begin{array}{l}1,290-2,271 \\
0,263-0,554\end{array}$ & $\begin{array}{l}7,786 \\
6,024\end{array}$ & $\begin{array}{l}1,87 * 10^{-6} \\
3,13 * 10^{-5}\end{array}$ \\
\hline
\end{tabular}

â : valeur estimée de l'ordonnée à l'origine $; \hat{b}$ : valeur estimée de la pente ; SE : erreur standard ; IC : intervalle de confiance ; $\mathrm{t}$ : statitique de Student ; p : probalilité

significatif au seuil de $5 \%(\mathrm{~F}=36,28 ; \mathrm{P}$ $\left.=3,12 * 10^{-5}\right)$. Le modèle obtenu est très robuste en ce sens que toutes les conditions liées aux résidus de régression ont été confirmées(Tableau 9). Ainsi, le test sur l'indépendance des résidus donne une statistique de Durbin-Watson comprise entre 1,5 et $3(\mathrm{DW}=2,81 ; \mathrm{P}=0,963)$. Ces résidus sont normalement distribués (statistique de Shapiro-Wilk : W $=0,943 ; \mathrm{P}=0,389$ ) et homogènes (statistique de Breush-Pagan: $\chi^{2}=$
0,027; $\mathrm{P}=0,870)$. La moyenne des résidus de régression est statistiquement proche de zéro $\left(\mathrm{m}=2,60 * 10^{-18} ; \mathrm{t}=3,87^{*} 10^{-17} ; \mathrm{P}=1\right)$. Par ailleurs, l'erreur standard résiduelle (ESR) du modèle est de 0,28 . La variation actuelle de l'abondance relative des espèces pourrait donc être expliquée par l'impact des engins et techniques de pêches (Tableau 8). De ce qui précède, l'équation de régression obtenue est:

$\operatorname{Ln}(A R)=1,780+0,409 \operatorname{Ln}(I I)\left(R^{2}=\right.$ $72,16 \%)$ 


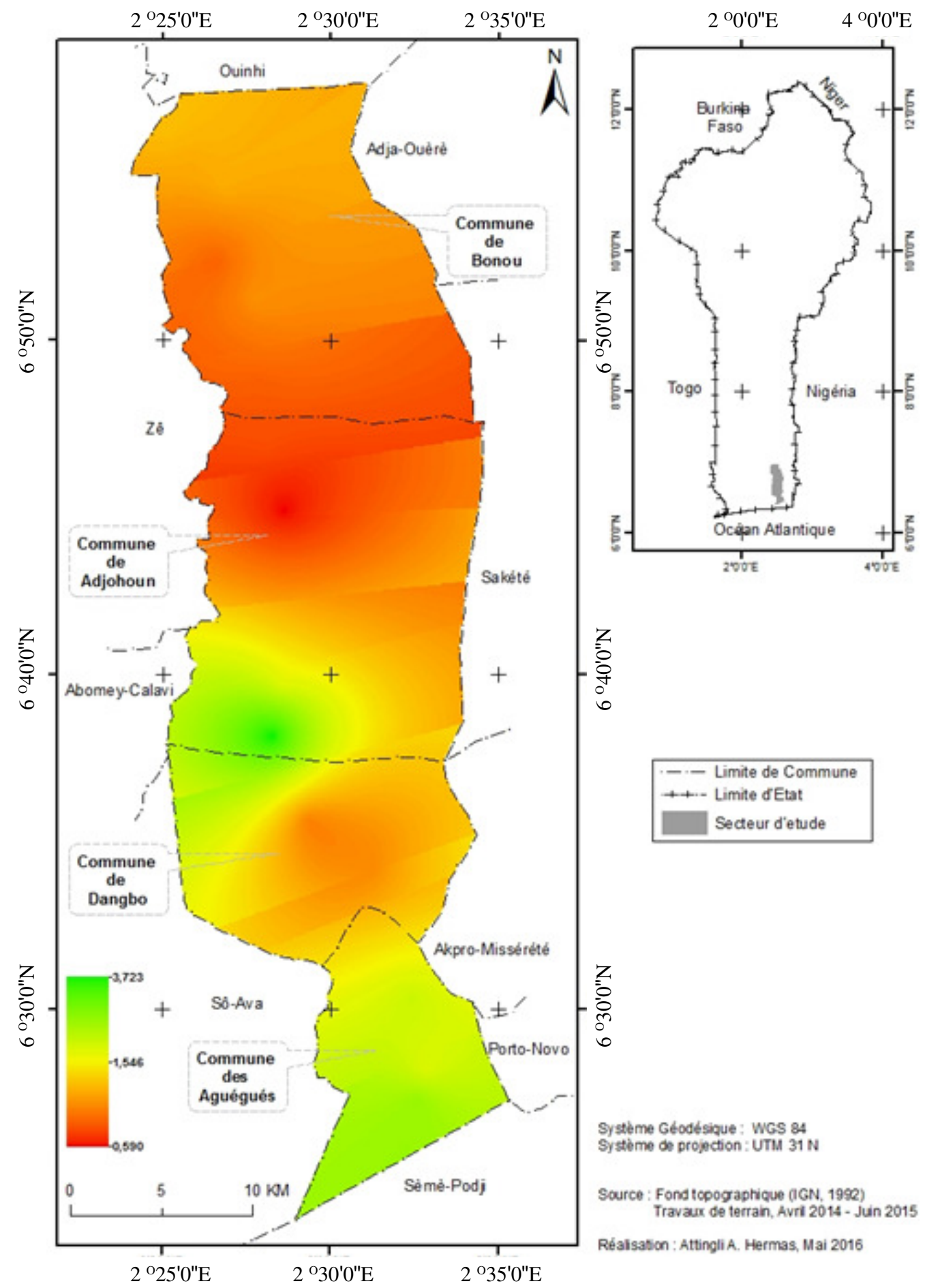

3.723 à 1.610. : Secteurs à forte abondance relative des espèces. 1.610 à 1.500 : secteurs à abondance relative moyenne des espèces. 1.500 à 0.590 : secteurs à faible à abondance relative des espèces

Figure 8. Spatialisation de l'abondance relative des espèces dans la BVO. 
L'analyse de l'équation montre que 72,20\% des variations actuelles de l'abondance relative du stock disponible sont expliquées par l'impact des engins et techniques de pêche dans la BVO. Pour un impact négligeable voire faible des engins et techniques de pêche de l'ordre d'une unité dans les zones de pêcherie, il en résulte une abondance relative de 6,16 unité. Ainsi, l'augmentation d'une unité supplémentaire de la valeur d'impact des engins et technique de pêche entrainerait une augmentation de l'abondance relative de 0,281. On note donc un accroissement de l'abondance relative du stock suite à une intensification des activités de pêche.

\section{DISCUSSION}

Les engins et techniques de pêche en exploitation dans la BVO impactent profondément sur le stock disponible eu égard à la composition de leur capture, leur nature et leur fréquence d'utilisation. Ainsi, les parcs à branchage «Acadja » et filets maillants qui se trouvent dans un même groupe de fréquence d'utilisation sont de nature plus dense en surface élémentaire et, sont souvent déployés sur de vastes étendues. Ce sont les techniques qui séjournent le plus longtemps dans les zones de pêcherie de la BVO. Le baril « Gbadja » et les Nasses de fréquence d'utilisation moins importante que les précédents ont un séjour relativement court. Quant aux filets épervier, la Palangre et «Dobah », ils sont des engins à faible fréquence d'utilisation. Les lignes, engin de pêche d'utilisation individuelle, sont de surface d'action faible et se pratiquent beaucoup sur les berges. Les études menées par Lalèyè et al. (2007) et Chikou (2006) vont dans le même sens. Ces auteurs ont présenté un inventaire exhaustif des engins et techniques de pêche en exploitation dans le secteur d'étude et les ont décrits. Parmi ceux-ci, les parcs à branchage «Acadja» sont plus cités. L'apparition du «Gbadja », nouvel engin de pêche, révèle une dynamique des pêcheurs dans le mode de sophistication des engins et techniques, orientée actuellement vers des prises aléatoires. Ceci s'expliquerait par la baisse progressive de rendement signalée par certains auteurs depuis quelques années (Welcomme, 1971; Lalèyè et al., 2007 ; Imorou Toko, 2007 ; Chikou, 2006). Le souci d'adaptation des activités de pêche aux changements globaux qui affectent les écosystèmes aquatiques a conduit les pêcheurs à une modification de l'ensemble de leur outillage dont les conséquences ont été souvent désastreuses :raréfaction d'espèces de grande taille, dégradation continue des écosystèmes aquatiques, forte concentration des dérivées nitreuses, prolifération de jacinthe d'eau (PNUD/FAO, 2000 ; Villanueva, 2004; Sala et al., 2008).

Si le besoin d'adaptation de l'outillage artisanale de pêche au contexte de rareté des prises dans la BVO a été souvent mis en évidence la force de nuisance de ces engins et technique de pêche sur le l'ichtyofaune n'est pas souvent bien connue. En effet, les huit (8) engins et techniquesrecensés ont des niveaux variables de nuisance, vue la composition de leur capture. Ces captures sont constituées en majorité de juvéniles et des individus de stade I, II et III qui devraient assurées le recrutement. Or, le stade III correspond à la période de reproduction des espèces (Baijot $e t$ al., 1994). Aussi sont-ils nombreux les individus de petites tailles que l'on y trouve. Les « Acadja » et filets maillants sont les engins et techniques de pêche dotés d'une force de nuisance plus destructrice sur le stock disponible car, ils capturent les espèces de toutes les tailles et de tous les stades de maturité critique pour le renouvellement du stock. Ces résultats rejoignent ceux de Chikou (2006) qui, avait déjà évoqué la capture des poissons en période de reproduction par les « Acadja » et filets maillants dans la BVO.

L'auteur signale également la prépondérance des individus de petites tailles dans les captures des filets maillant de mailles inférieures ou égales à $15 \mathrm{~mm}$.Abondant dans le même sens, Lalèyè et al. (2007) a indiqué que les captures 
des filets maillants sont dominées par des espèces de poissons de petites tailles dont Petrocephalus bovei (15,5 \%), Schilbe intermedius (14,3\%) et Synodontis schall $(10,1 \%)$. Ahouansou Montcho(2011) avait fait observer que dans les parcs à branchages «Acadja », la taille des poissons varie entre 4,8 et $23,8 \mathrm{~cm}$. Quant aux « Dobah », Nasses et Filets éperviers, ils présentent une force de nuisance moyenne. En effet, le «Dobah», ne semble pas être très actif dans la capture des espèces de petite taille comme les nasses. Le «Dobah » et la nasse ont un caractère saisonnier d'exploitation dans le secteur d'étude. Les filets éperviers, d'utilisation faible (principalement en aval de la BVO dans la Commune des Aguégués), revêtent également une force de nuisance moyenne pour la biodiversité ichtyologique.

Les «Gbadja », Palangres et lignes, sont des engins et techniques de pêche de faible nuisance non pas parce qu'ils ne capturent pas les espèces en stade de maturité critique ou de petite taille, mais parce qu'ils ont la particularité d'avoir une empreinte spatiale faible dans les zones de pêcheries de la BVO. De plus, leurs captures sont de composition très peu variable et moins pondérée. Le« Gbadja » est conçu en effet, pour capturer dans la pratique uniquement les Chrysichthys.

Globalement sur les huit engins et techniques, le filet épervier, la nasse, le Dobah, la palangre et la ligne peuvent être encore recommandée pour l'exploitation de pêche dans la BVO. Mais le Gbadja n'est pas recommandable, car il tient son caractère de faible nuisance à son exploitation localisée. En effet, cette technique est en réalité très nuisible de l'ichtyofaune parce qu'elle est très sélective. Sa généralisation à la $\mathrm{BVO}$ serait donc suicidaire pour l'équilibre de l'écosystème. Le parc à branchage « Acadja » de par sa nature contribue à la pollution des plans d'eau, car son efficacité relève de la densité des branchages.

Lalèyè et al. (2003) avait indiqué que les rendements des acadja variaient de 1,5 à 15,5 t ha ${ }^{-1} a^{-1}$ en fonction de la densité des branchages et de l'efficacité de la pêche. Or, la décomposition de ces branchages au contact de l'eau provoque des réactions chimiques. Ce qui pourrait renforcer la concentration des polluants organiques responsables de la pollution organique rapportée par YéhouénouPazou (2005) et Zinsou et al. (2016) dans la BVO.

L'impact des engins et techniques de pêche analysé dans la présente étude est une pondération linéaire des captures effectuées dans l'ensemble des zones de pêcherie du secteur d'étude. Il complète l'indice de nuisance en apportant l'occurrence des espèces par capture quelle que soit la catégorie de variable considérée sur l'ichtyofaune. Ainsi, dans le secteur d'étude, on note un gradient décroissant de l'impact des engins et techniques de pêche sur l'ichtyofaune des pêcheries de l'aval à celles de l'amont. Les zones de pêcherie située en aval de la BVO sont celles qui subissent le plus les assauts répétés et continuels des engins et techniques de pêches.

La spatialisation de la tendance a permis de mieux appréhender le phénomène à l'échelle communale. Ainsi, La Commune des Aguégués, les parties Sud et Ouest de la Commune de Dangbo et le Sud-ouest de la Commune d'Adjohoun en sont les plus affectées. Ceci s'expliquerait par le fait que c'est dans ces parties du secteur d'étude que l'activité de pêche s'exerce encore avec plus d'intensité. En effet, les populations riveraines (xwla, Tofin et Wémè) ayant une grande tradition de pêche (Gbaguidi, 2009) sont restées fidèles à cette activité en dépit des nouvelles contraintes. Aussi, est-il important de souligner la forte demande en produits de pêche due à la proximité des grandes agglomérations de Cotonou, sèmè kpodji et Porto-Novo à la BVO.

Dans le secteur d'étude, l'abondance relative des espèces suit le même gradient que celui précédemment indiqué pour l'impact des engins et techniques de pêche. Les zones de pêcherie situées en Aval de la BVO apparaissent donc potentiellement plus diversifiées que 
celles situées en amont. L'existence d'une corrélation a conduit à la construction d'un modèle de prédiction dont les conditions de validité semblent confirmé une relation de type puissance entre l'abondance relative des espèces et la valeur d'impact des engins et technique de pêche. En effet, plus la pression des engins et techniques est intensifiée dans une pêcherie, plus les captures sont diversifiées. Cet état de fait, bien que anodine, montre que les pressions de pêche provoqueraient toutefois une recolonisation des zones de pêcheries et périphéries par des espèces allochtones. Villanueva (2004) dans ces travaux sur le Lac Nokoué avait souligné les incursions saisonnières d'espèces entre deux écosystèmes adjacents se combinant avec la présence permanente d'une communauté typiquement estuarienne. C'est pourquoi bien que la BVO soit en eau douce, la liste des poissons recensés révèle la présence d'espèces marines et lagunaires (Chikou, 2006).

La BVO est confrontée à des difficultés de gestion, au point où les espèces de grandes tailles telles que Heterobranchus longifilis, Gymnarchus niloticus, lates niloticussous l'effet des pratiques de pêche sont devenues plus rares (Lalèyè et al., 2007), et, ont laissé place aux poissons-chats plus tolérants aux conditions hypoxiques actuelles du milieu(Imorou Toko, 2007). Face à une telle situation, une sélection des engins et techniques moins nuisibles aux conditions de renouvèlement des stocks peut contribuer à moyen ou long terme à la restauration des plans et cours d'eau aujourd'hui plus que par le passé sous forçages climatiques. Les prises accessoires, définies comme les espèces non cibles pour l'activité de pêche pourraient être protégées dans les cas extrêmes.

\section{CONCLUSION}

L'ichtyofaune de la Basse Vallée de l'Ouémé est fortement influencée par les engins et techniques de pêche utilisés par les populations riveraines. Ces engins et techniques présentent, non seulement, de forte nuisance mais leur fréquence d'utilisation et les catégories d'espèces capturées en font des outils à fort impact sur l'ichtyofaune surtout dans la partie aval du secteur d'étude. Il convient donc d'élaborer un plan d'aménagement dans laBVO qui intègre une règlementation des engins et techniques de pêche limités strictement à ceux qui ont une emprise moins contrayantes pour l'ichtyofaune. Cela pourra contribuer à la restauration de l'immense potentialité de cet écosystème. De plus, l'organisation des pêcheurs, la sensibilisation et la recherche interactive des engins et techniques adéquats pour une gestion durable de la ressource seraient des pistes utiles pour la réussite et la mise en place du plan d'aménagement.

\section{REMERCIEMENT}

Les auteurs adressent leur remerciement au Projet de Productivité Agricole en Afrique de l'Ouest (PPAAO) du Programme Cadre d'Appui à la diversification agricole (ProCAD) du Ministère de l'Agriculture et de la Pêche (MAEP) au Bénin.

\section{RÉFÉRENCES BIBLIOGRAPHIQUES}

Ahouansou Montcho, S., Lonhodé, JDS., Akochéou, AGG., Tokpanou, CF., Lédéroun D. et Lalèyè P. 2016. Détermination de maillage de filets pour une exploitation durable de Saritherodon galilaeus à la retenue d'eau Gougan de Kogbétohoué au Bénin. Bulletin de la recherche Agronomique du Bénin (BRAB). Numéro spécial Agronomie, Société, Environnement et Sécurité Alimentaire. pp. 85-92.

Ahouansou Montcho, S. 2011. Diversité et exploitation des poissons de la rivière Pendjari (Bénin, Afrique de l'Ouest). Thèse de doctorat, Université d'Abomey-Calavi, Bénin. 234 pp.

Attingli, A.H., Zinsou H.L., Vissin W.E. et Lalèyè P. 2016. Spatialisation des 
paramètres physico-chimiques dans les pêcheries de la Basse Vallée de l'Ouémé. (sud-Bénin). Journal of Applied Biosciences 105:10190-10202.

Baijot, E., Moreau, J. et Bouda, S. 1994. Aspects hydrobiologiques et piscicoles des retenues en zone soudano-sahélienne. CTA, Wageningen. $250 \mathrm{pp}$.

Balirwa, J.S., Chapman, C.A., Chapman, L.J., Cowx, I.G., Geheb, K., Kaufman, L. and Witte, F. 2003. Biodiversity and fishery sustainability in the Lake Victoria basin: an unexpected marriage. BioScience 53(8): 703-716.

Brown-Peterson, N.J., Wyanski, D.M., Saborido-Rey, F., Macewicz, B.J. and Lowerre-Barbieri, S.K. 2011. A standardized terminology for describing reproductive development in fishes. Marine and Coastal Fisheries 3(1):52-70.

Chikou, A. 2006. Etude de la démographie et de l'exploitation halieutique de six espèces de poissons-chats (Teleostei, Siluriformes) dans le delta de l'Ouémé au Bénin. Thèse de Doctorat, Université de Liège, Belgique, $417 \mathrm{pp}$.

Dagnelie, P. 1973. Théorie et méthodes statistiques. Applications Agronomiques. Vol. 1. Presses agronomiques de Gembloux. 378 pp.

Direction des pêches (DP), 2008. Données enquête cadre : Pêche continentale et aquaculture au plan national. Rapport final, Cotonou, Bénin. 89 pp.

FAO, 2006. Profil des pêches et de l'aquaculture par pays : vue générale du secteur des pêches nationales (Afrique de l'Ouest). FAO/FIR/CP/BEN, FAO, Rome, Italie. $42 \mathrm{pp}$.

FAO, 2011. Directives internationales sur la gestion des prises accessoires et la réduction des rejets en mer. $73 \mathrm{pp}$.

Gbaguidi, A. 2009. Enquête-cadre sur la pêche continentale et l'Aquaculture PADPPA/ CBRST Bénin. 82 pp.

Imorou Toko, I. 2007. Amélioration de la production halieutique des trous traditionnels à poissons «whédo» du Delta de l'Ouémé (Sud Bénin) par la promotion de l'élevage des poissons-chats Clarias gariepinus et Heterobranchus longifilis. Thèse de Doctorat, Facultés universitaires Notre-Dame de la Paix-Namur, Belgique. $186 \mathrm{pp}$.

INSAE, 2013. Résultats provisoires du RGPH4. Institut National de la Statistique et de l'Analyse Economique, Cotonou, Bénin. 8 pp.

Kantoussan, J. 2007. Impacts de la pression de pêche sur l'organisation des peuplements de poissons: Application aux retenues artificielles de Sélingué et de Manantali, Mali, Afrique de l'Ouest. Thèse de doctorat, Agrocampus, Rennes. 195 pp.

Laë, R. 1992. Influence de l'hydrologie sur l'évolution des pêcheries du delta central du Niger, de 1966 à 1989. Aquatic Living Resources 5(2):115-126.

Lalèyè, P. A. 1995. Ecologie comparée de deux espèces de Chrisichthys, poissons siluriformes (Claroteidae) du complexe lagunaire lac Nokoué-lagune de Porto-Novo au Bénin. Thèse de Doctorat en Sciences, Université de Liège, Belgique. 152 pp.

Lalèyè, A.P., Chikou, A., Philippart, J-C., Teugels, G. et Vandewalle, P. 2004. Étude de la diversité ichtyologique du bassin du fleuve Ouémé au Bénin (Afrique de l'Ouest). Cybium 28(4):329-339.

Lalèyè, A.P., Ezin, A., Vandewalle, P., Phillippart, J-C and Teugels, G.G. 2007. Caractéristiques de la pêche dans le fleuve Ouémé au Bénin (Afrique de l'Ouest). Journal. Afrotrop. Zoology. Special issue : 137-148

Ministère de l'Agriculture, de l'Elevage et de la Pêche(MAEP), 2009. Statistiques Direction des Pêches, Cotonou, Bénin. $57 \mathrm{pp}$.

Murawski, S.A. 2000. Definitions of overfishing from an ecosystem perspective. ICES Journal of Marine, Science 57:649-658. 
Paugy, D., Lévèque, C. et Teugels, G.G. 2004. Faune des poissons d'eau douce et saumâtres de l'Afrique de l'Ouest. Publications Scientifiques du Muséum. Ed. IRD. MRAC, Belgique.

Lalèyè, Ph., Chikou, A., Ezin, A., Philippart, J.C., Welcomme, R.L. 2003. Fish and fisheries of the Oueme Delta, Benin (West Africa). Paper presented at the Second International Symposium on the Management of large Rivers for Fisheries, Phnom Penh, 2003.

Plisnier, P.D. 1990. Ecologie compare et exploitation rationnelle de deux populations d'haplochromis spp. (teleostei Cichlidae) des lacs Ihema et Muhazi (Rwanda). Thèse de doctorat en Sciences Agronomiques. Université Catholique de Louvain. 324 pp.

PNUD/FAO, 2000. Rapport du Consultant National sur Pêches Continentales et Aquaculture. République du Bénin. Ministére du Développement Rural. Projet PNUD/FAO SPPD-BEN/99/004. 43pp.

Scheffe, H. 1959. The Analysis of Variance. New York, USA. pp. 331-367.

Sohou, Z., Houedjissin, R.C. et Ahoyo, N.RA. 2009. La pisciculture: de la tradition à la modernisation. Bulletin de Recherche
Agronomique du Bénin. Numéro 66. Décembre 2009.

Villanueva, M.C.S. 2004. Biodiversité et relations trophiques dans quelques milieux estuariens et lagunaires de l'Afrique de l'Ouest. Adaptations aux pressions environnementales. Thèse de doctorat. INPT/ENAST/Toulouse. 246 p. + résumé.

Welcomme, R.L. 1971. Evaluation de la pêche intérieure, son état actuel et ses possibilités. FAO, AT 2938, Rome, Italy. 95 pp.

Worm, B., Barbier, E.B., Beaumont, N., Duffy, J.E., Folke, C., Halpern, B.S. and Sala, E. 2006. Impacts of biodiversity loss on ocean ecosystem services. Science 314(5800): 787-790.

Yehouenou-Pazou, A.E. 2005. Les résidus de pesticides chimiques de synthèse dans les eaux, les sédiments et les espèces aquatiques du bassin versant du fleuve Ouémé et du lac Nokoué. Thèse de Doctorat unique. Université d'Abomey Calavi, Bénin 217pp.

Zinsou, H.L., Attingli, A.H., Gnohossou, P., Adandedjan, D. et Laleye P. 2016. Caractéristiques physico-chimiques et pollution de l'eau du delta de l'Ouémé au Bénin. Journal of Applied Biosciences 97:9163-9173. 